\title{
The role of photodynamic therapy in the management of oral dysplasia
}

\author{
Colin Hopper ${ }^{*}$, Waseem Jerjes, Zaid Hamdoon, Tahwinder Upile \\ From 2nd Scientific Meeting of the Head and Neck Optical Diagnostics Society \\ San Francisco, CA, USA. 23-24 January 2010
}

\section{Introduction}

Photodynamic therapy (PDT) is a minimally invasive surgical intervention used in the management of tissue disorders. It can be applied before, or after, any of the conventional modalities, without compromising these treatments or being compromised itself. PDT is valuable for potentially malignant disorders.

\section{Materials and methods}

In this prospective study, a total of 147 consecutive patients with potentially malignant disorders were treated with surface illumination PDT, using 5-ALA or mTHPC as the photosensitiser. The average age was $53 \pm 8.9$ years. Patients' recovery was uneventful and no complications reported. Comparisons with the clinical and histopathological features and rate of recurrence as well as malignant transformation were made. These patients were followed-up for a mean of 7.3 years, and biopsies taken in case of changes indicative of malignant development.

\section{Analysis and results}

The results were analysed by an independent statistician using SPSS 17. The results were cross tabulated and the Chi-squared statistic was used to test for differences in the case-mix.

Homogenous leukoplakias were identified in 55 patients, non-homogenous leukoplakias in 73 patients, whereas 19 patients had erythroplakias. Ex- and current lifelong smokers formed $84.4 \%$ of the recruited patients. While people who currently smoke and drink formed 38.1\% (56 patients) of the cohort. Erythroplakias were mainly identified in heavy lifelong smokers. The most common identified primary anatomical locations were the lateral border of tongue, floor of mouth and retromolar area. Moderate dysplasia was identified in 33 patients while 63 patients had severe dysplasias; and 32 patients had a histopathological diagnosis of carcinoma in situ.

The rate of first recurrence in laser surgery was approximately $11.6 \%$. The rate of recurrence had no significant association with the location or the severity of epithelial dysplasia. Malignant transformation was observed in 11 patients (7.5\%), in the tongue, floor of mouth and retromolar area. Recurrence and malignant transformation was mainly identified in erythroplakias and non-homogenous leukoplakias. The final outcome of the cohort showed that $11(7.5 \%)$ suffered from progressive disease, 5 (3.4\%) has stable disease, $12(8.2 \%)$ were considered partially responsive to the therapy. Complete response was identified in 119/147 patients (81\%).

\section{Conclusion}

5-ALA-PDT and/or mTHPC-PDT offer an effective alternative treatment for potentially malignant oral disorders. It is associated with excellent functional and cosmetic results and can be used in conjunction with other standard therapies.

Published: 29 October 2010

doi:10.1186/1758-3284-2-S1-024

Cite this article as: Hopper et al:: The role of photodynamic therapy in the management of oral dysplasia. Head \& Neck Oncology 2010 2(Suppl 1):O24. 